'Проблемная научно-исследовательская лаборатория «Диагностические исследования и малоинвазивные технологии», ФГБОУ ВО «Смоленский государственный медицинский университет» Минздрава РФ, Смоленск, Россия ${ }^{2}$ Кафедра лучевой диагностики и лучевой терапии, ФГБОУ ВО «Смоленский государственный медицинский университет» Минздрава РФ, Смоленск, Россия

ОБОСНОВАНИЕ. Благодаря доступности, высокой информативности и безвредности ультразвуковое исследование (УЗИ) является крайне эффективным методом дифференциальной диагностики патологии щитовидной железы (ЩЖ). Несмотря на достаточный арсенал диагностических методов, биопсия ЩЖ с цитологическим исследованием является золотым стандартом в диагностических алгоритмах. Наиболее часто тонкоигольную аспирационную биопсию (ТАБ) осуществляют с использованием одноразовых шприцев разной емкости $\left(5,10,20\right.$ мл ${ }^{3}$. Выполняют процедуру в большинстве случаев по методике Free hand. Это приводит сразу к нескольким проблемам, связанным с особенностями выполнения исследования, в первую очередь с создаваемым низким уровнем вакуума в шприце, недостаточным забором цитологического материала и недостаточным практическим опытом у начинающего специалиста.

ЦЕЛЬ. Определение диапазона возможности использования вакуумной ТАБ под контролем ультразвуковой навигации у пациентов с очаговой патологией щЖ.

МАТЕРИАЛЫ И МЕТОДЫ. На базе Проблемной научно-исследовательской лаборатории «Диагностические исследования и малоинвазивные технологии» ФГБОУ ВО СГМУ Минздрава РФ было разработано оригинальное устройство для вакуумной ТАБ (v-ТАБ). В период 2019-2020 гг. было выполнено 780 v-ТАБ под контролем ультразвуковой навигации пациентам с узловыми образованиями ЩЖ. Уровень разрежения в шприце во время забора материала составил 0,5 bar $(-50 \mathrm{kPa})$.

РЕЗУЛЬТАТЫ. При сравнительном анализе цитологических результатов, полученных при помощи v-ТАБ и методики Free hand, наблюдается уменьшение категории Bethesda 1 на 3,3\% и Bethesda 3 на 10,2\% у пациентов с очаговой патологией щЖ.

\title{
ЗАКЛЮЧЕНИЕ.
}

1. v-ТАБ по сравнению со стандартной методикой Free hand обладает большей информативностью в диагностике очаговой патологии ЩЖ.

2. За счет постоянного визуального контроля и отсутствия движения поршня шприца во время проведения v-ТАБ снижается вероятность отклонения иглы от зоны биопсии, что повышает эффективность исследования и облегчает работу начинающему специалисту.

КЛЮЧЕВЫЕ СЛОВА: вакуум-пункция; ультразвуковая навигация; узловой зоб.

\section{EXPERIENCE OF USING VACUUM FINE-NEEDLE ASPIRATION BIOPSY UNDER ULTRASOUND CONTROL IN PATIENTS WITH FOCAL THYROID PATHOLOGY}

\author{
(c) Alexey V. Borsukov', Anton O. Tagil2*
}

'Problem scientific research laboratory «Diagnostic researches and minimally invasive technologies», Smolensk State Medical University, Smolensk

${ }^{2}$ The Department of radiology and radiation therapy, Smolensk State Medical University, 214006, Smolensk, Russia

BACKGROUND: Due to its availability, high information content and harmlessness, ultrasound is an extremely effective method of differential diagnosis of thyroid pathology. Despite the sufficient arsenal of diagnostic methods, thyroid biopsy with cytological examination is the «gold» standard in diagnostic algorithms. Most often, fine - needle aspiration biopsy is carried out using disposable syringes of different capacities $(5,10,20 \mathrm{ml})$. The procedure is performed in most cases according to the «Free hand» method, this leads to several problems associated with the peculiarity of the study, primarily with the low vacuum created in the syringe and insufficient collection of cytological material, as well as insufficient practical experience for a novice specialist. 
AIM: Determination of the range of the possibility of using vacuum aspiration fine needle biopsy under the control of ultrasound navigation in patients with focal thyroid pathology.

MATERIALS AND METHODS: On the basis of the Problem Research Laboratory «Diagnostic studies and minimally invasive technologies» of the Smolensk State Medical University, a device for vacuum fine - needle aspiration biopsy was developed. In the period 2019-2020, 780 vacuum fine - needle aspiration biopsy was performed under the control of ultrasound navigation in patients with thyroid nodules. The level of vacuum in the syringe during sampling of the material was $0,5$ bar (- $50 \mathrm{kPa})$.

RESULTS: A comparative analysis of the cytological results obtained with the help of vacuum fine - needle aspiration biopsy and the «Free hand» method shows a decrease in obtaining the category of Bethesda 1 by $3.3 \%$ and Bethesda 3 by $10.2 \%$.

CONCLUSION: This indicates the effectiveness and in formativeness of the use of vacuum fine - needle aspiration biopsy in patients with focal thyroid pathology in comparison with the standard «Free hand» method, as well as with vacuum fine - needle aspiration biopsy due to constant visual monitoring and the lack of movement of the syringe piston to create a discharge when taking cytological material, the probability of needle deviation from the biopsy zone is reduced, which increases the effectiveness of the study and facilitates the work of a novice specialist.

KEYWORDS: vacuum puncture; ultrasound navigation; nodular goiter.

\section{ОБОСНОВАНИЕ}

Одними из основных методов диагностики, используемых при заболеваниях щитовидной железы (ЩЖ), являются: пальпация ЩЖ и лимфатических узлов, определение уровня тиреоидных и тиреотропных гормонов и ультразвуковое исследование (УЗИ). Благодаря доступности, высокой информативности и безвредности УзИ является крайне эффективным методом дифференциальной диагностики патологии ЩЖ. Несмотря на достаточный арсенал диагностических методов, биопсия ЩЖ с цитологическим исследованием является золотым стандартом в диагностических алгоритмах [1-3].

Использование методов ультразвуковой навигации для выполнения малоинвазивных вмешательств значительно облегчает манипуляцию и снижает риск развития возможных осложнений, но не исключает возможность их появления [4, 5].

Однако, независимо от ценности метода, существующие зарубежные и российские исследования указывают на возможность наличия погрешности в интерпретации результатов. Возможность получения ложноположительных результатов рака ЩЖ составляет 0-7\%, а ложноотрицательных - находится в диапазоне 1-11\% [6]. Чувствительность и специфичность метода также имеют достаточно широкие пределы $[7,8]$.

Наиболее часто тонкоигольную аспирационную биопсию (ТАБ) осуществляют с использованием одноразовых шприцев разной емкости $\left(5,10,20\right.$ мл $\left.{ }^{3}\right)$. В большинстве случаев реализация данного исследования производится по методике Free hand [3, 9], выполнение которой подразумевает одновременное использование обеих рук в момент забора цитологического материала при условии выполнения процедуры одним специалистом. Это приводит к слепому забору цитологического материала, что может способствовать непроизвольному смещению иглы в сторону важных анатомических структур, а при наличии двух специалистов (врача ультразвуковой диагностики и врача, выполняющего процедуру) возможна неправильная визуализация иглы из-за отсутствия синхронности в работе специалистов. Кроме того, разная плотность узловых образований в ЩЖ и непра- вильно подобранный объем шприца могут привести к «сухой биопсии», то есть недостаточному забору материала и неинформативному результату цитологического исследования [10].

В связи с имеющимися особенностями проведения ТАБ существует методика, которая позволяет регулировать уровень разрежения в шприце и предоставляет возможность для одновременного проведения биопсии и визуального ультразвукового контроля за ее проведением одному специалисту, при использовании вакуумной ТАБ (v-ТАБ). Примером для разработки послужило устройство Трошина В.П. и соавт., патент R58025UU1 [11].

Физическое обоснование вакуум-биопсии основано на том, что шприц объемом 5 мл ${ }^{3}$ создает отрицательное давление, равное -0,1 bar (-10 kPa), 10 мл म $^{3}-0,21 \mathrm{bar}$ (-21 kPa), 20 мл - $^{3}$-0,35 bar (-35 kPa), шприц Жане объемом 160 мл ${ }^{3}$ создает давление -0,83 bar (-83 kPa). В практической деятельности для ТАБ обычно используются шприцы объемом 10 и 20 мл ${ }^{3}$, в зависимости от очагового образования и анатомического размера кисти специалиста, выполняющего манипуляцию, так как 20-миллилитровый шприц не всегда удобен.

В настоящее время нет данных об эффективности использования v-ТАБ по сравнению со стандартной ТАБ, а также об изменении спектра цитологических зон исследования по Bethesda после применения v-ТАБ.

\section{ЦЕЛЬ ИССЛЕДОВАНИЯ}

Определение диапазона возможности использования v-ТАБ под контролем ультразвуковой навигации у пациентов с очаговой патологией щЖ.

\section{МАТЕРИАЛЫ И МЕТОДЫ}

\section{Дизайн исследования:}

- одноцентровое;

- экспериментальное с медицинскими вмешательствами;

- динамическое, проспективное;

- одновыборочное контролируемое рандомизированное. 


\section{МЕТОДЫ}

На базе Проблемной научно-исследовательской лаборатории «Диагностические исследования и малоинвазивные технологии» Федерального государственного бюджетного образовательного учреждения высшего образования «Смоленский государственный медицинский университет» Минздрава РФ в период с 2014 по 2019 гг. было выполнено 3247 ТАБ пациентам с очаговой патологией щЖ. Пункционную биопсию выполняли под ультразвуковым контролем аппарата Fujifilm Sonosite Edge, оснащенного линейным датчиком HFL50x частотой 15-6 МГц. Пункция проводилась иглой $21 \mathrm{G}$ длиной 4 см в комплекте с 10-миллилитровым шприцом методикой Free hand. Пунктировали все узловые образования размером более 10 мм, а также узловые образования меньшего размера, но имеющие подозрительныеУЗ-признаки (TIRADS 4A и более) или высокие показатели лабораторных данных (уровень базального или стимулированного кальцитонина >100 пг/мл), в соответствии с клиническими рекомендациями Российской ассоциации эндокринологов по диагностике и лечению (много)узлового зоба у взрослых 2016 г. [2, 12]. Весь пункционный материал помещался на 4 обезжиренных предметных стекла, после чего приготавливались тонкие мазки и отправлялись на цитологическое исследование с последующей фиксацией материала (табл. 1) и дальнейшей интерпретацией результатов по классификации Bethesda 2009 [13-15].

Наибольший интерес составляют пациенты категорий I и III по Bethesda, поскольку именно этой группе лиц необходимо повторное проведение ТАБ для получения более информативных результатов и определения дальнейших действий по отношению к имеющейся патологии. Поскольку от морфологической структуры и анатомического расположения узлового образования в доле ЩЖ (близкое расположение к общей сонной артерии или внутренней яремной вене), а также его размера зависит количество полученного материала, на базе Проблемной научно-исследовательской лаборатории «Диагностические исследования и малоинвазивные технологии» Смоленского государственного медицинского

Таблица 1. Цитологическое заключение по классификации Bethesda 2009 при тонкоигольной аспирационной биопсии по методике Free hand (Смоленск 2013-2019 гг.)

\begin{tabular}{|c|c|c|}
\hline \multicolumn{2}{|c|}{ Цитологическое заключение } & \multirow{2}{*}{$\begin{array}{c}\text { Пациенты, } \\
\text { n (\%) } \\
208(6,4)\end{array}$} \\
\hline Bethesda I & $\begin{array}{l}\text { Неинформативная } \\
\text { пункция }\end{array}$ & \\
\hline Bethesda II & $\begin{array}{l}\text { Доброкачественное } \\
\text { образование }\end{array}$ & $1993(61,4)$ \\
\hline Bethesda III & $\begin{array}{l}\text { Атипия неопределенного } \\
\text { значения }\end{array}$ & $572(17,6)$ \\
\hline Bethesda IV & Фолликулярная неоплазия & $318(9,8)$ \\
\hline Bethesda V & $\begin{array}{l}\text { Подозрение на } \\
\text { злокачественную опухоль }\end{array}$ & $114(3,5)$ \\
\hline Bethesda VI & Злокачественная опухоль & $42(1,3)$ \\
\hline
\end{tabular}

университета было разработано оригинальное устройство для v-ТАБ, заявка на оригинальное изобретение №2020137115/20, позволяющее одному специалисту выполнять забор цитологического материала с одновременным визуальным ультразвуковым контролем расположения иглы в зоне биопсии, что снижает возможность дислокации иглы и развитие осложнений.

\section{Статистический анализ}

Статистическая обработка материала проводилась с помощью пакета Statistica 7.0, SPSS 10.

\section{Этическая экспертиза}

Локальный этический комитет (ЛЭК) на базе ОГБУЗ «Клиническая больница №1» города Смоленска. Протокол № 23 от 29.09.2019 г.

\section{РЕЗУЛЬТАТЫ}

В период 2019-2020 гг. было выполнено 780 v-ТАБ пациентам с узловыми образованиями щЖ (табл. 2). Пациенты были направлены на исследование участковыми врачами-эндокринологами и хирургами на базу ПНИЛ в городское отделение диагностических и малоинвазивных технологий ОГБУЗ «Клиническая больница №1» города Смоленска. Большинство узловых образований носило доброкачественный характер (Bethesda II), однако у 24 $(3,1 \%)$ пациентов результат оказался неинформативным (Bethesda I), что связано с избыточным количеством элементов крови в полученном цитологическом материале.

При сравнительном анализе цитологических результатов, полученных при помощи v-ТАБ и методики Free hand, наблюдается снижение получения результатов категории Bethesda I на 3,3\% и Bethesda III на 10,2\% (табл. 2). Также при анализе таблицы 2 отмечено отсутствие пациентов категории Bethesda VI, что связано с ограниченным поступлением пациентов в ОГБУЗ «Клиническая больница №1» из-за мер, обусловленных пандемией COVID-19.

Пациентам I и III категорий по Bethesda, которым проводилась v-ТАБ, было выполнено повторное исследование через определенный промежуток времени,

Таблица 2. Соотношение полученных цитологических результатов вакуумной тонкоигольной аспирационной биопсии и Free hand по Bethesda 2009, n (\%)

\begin{tabular}{lcc}
\hline $\begin{array}{c}\text { Kатегория } \\
\text { Bethesda }\end{array}$ & $\begin{array}{c}\text { V-TAБ } \\
(\mathbf{2 0 1 9 - 2 0 2 0 )}\end{array}$ & $\begin{array}{c}\text { Free hand } \\
\mathbf{( 2 0 1 4 - 2 0 2 0 )}\end{array}$ \\
\hline Bethesda I & $24(3,1 \%)$ & $208(6,4 \%)$ \\
Bethesda II & $618(79,2 \%)$ & $1993(61,4 \%)$ \\
Bethesda III & $58(7,4 \%)$ & $572(17,6 \%)$ \\
Bethesda IV & $68(8,8 \%)$ & $318(9,8 \%)$ \\
Bethesda V & $12(1,5 \%)$ & $114(3,5 \%)$ \\
Bethesda VI & - & $42(1,3 \%)$ \\
\hline
\end{tabular}


Таблица 3. Цитологические заключения при повторной вакуумной тонкоигольной аспирационной биопсии, $\mathrm{n}(\%)$

\begin{tabular}{lc}
\hline \multicolumn{1}{c}{$\begin{array}{c}\text { Kатегория } \\
\text { Bethesda }\end{array}$} & $\begin{array}{c}\text { V-тАБ } \\
(\mathbf{2 0 1 9 - 2 0 2 0 )}\end{array}$ \\
\hline Bethesda I & $8(1,02 \%)$ \\
Bethesda II & $54(6,9 \%)$ \\
Bethesda III & $11(1,4 \%)$ \\
Bethesda IV & $6(0,77 \%)$ \\
Bethesda V & $3(0,38 \%)$ \\
Bethesda VI & $2(0,26 \%)$ \\
\hline
\end{tabular}

но с разным уровнем разрежения в шприце, который зависел от ультразвуковой семиотики узлового образования с отсутствием крупных сосудов в зоне забора цитологического материала, по данным цветного допплеровского картирования (ЦДК), и избеганием жидкостного компонента с приоритетом на эластографическую неоднородность и жесткие зоны очага по данным эластографии в режиме SE и SWE $[16,17]$. У большей части пациентов повторный результат оказался информативным (табл. 3).

Например, при наличии, по данным В-режима, УЗ-признаков аденоматозного образования (округлая форма с четкими ровными контурами, достаточно однородной структуры, имеющей гипоэхогенный ободок) и кровотока по ЦДК с неоднородными участками в очаге, по данным эластографии, в связи с избыточной васкуляризацией образования уровень разрежения в шприце снижался до -0,3 bar (-30 kPa), что предотвращало избыточный забор элементов крови с определением в мазке тубулоподобных с концентрическим формированием тироцитов клеток, с наличием в центральной части коллоидной субстанции, встречающихся при фолликулярной аденоме.

Это указывает на эффективность и информативность использования v-ТАБ у пациентов с очаговой патологией ЩЖ по сравнению со стандартной методикой Free hand.

Дополнительно подтверждает полученные результаты проведенное на базе Проблемной научно-исследовательской лаборатории «Диагностические исследования и малоинвазивные технологии» в период с 2016 по 2018 гг. исследование, в котором определялась амплитуда движения и отклонения иглы в зоне биопсии в зависимости от практического опыта врача (табл. 4). Суть исследования заключалась в тренировке врачей ТАБ по методике Free hand на разработанном фантоме.

При анализе полученных результатов выяснилось, что в зависимости от опыта специалиста определяется корреляция погрешности движений иглы в зоне забора цитологического материала в нескольких плоскостях. Это приводит к выводу, что у начинающего специалиста уровень информативности исследования снижается за счет недостаточной амплитуды движения иглы в зоне биопсии, из-за чего не происходит достаточного забора клеточного материала на фоне травматизации паренхимы ЩЖ, что приводит к необходимости повторного исследования через определенный промежуток времени. Все указанные аспекты задерживают дальнейший диагностический и лечебный алгоритм как со стороны лечащего врача, так и со стороны пациента, что вынуждает придерживаться выжидательной тактики, которая может негативно отразиться на состоянии пациента до проведения повторного исследования, которое также не гарантирует получения информативного результата. Соответственно, при v-ТАБ изначально за счет постоянного визуального контроля и отсутствия движения поршня шприца для создания разрежения снижается вероятность отклонения иглы от зоны биопсии, что повышает эффективность исследования и облегчает работу начинающему специалисту.

Клиническая значимость результатов

Применение v-ТАБ в клинической практике снижает риск развития осложнений в момент проведения манипуляции и повышает информативность по сравнению со стандартной методикой Free hand.

Направления дальнейших исследований

Увеличение количества выборки пациентов с целью индивидуализации уровня отрицательного давления в шприце для забора цитологического материала в зависимости от ультразвуковой и эластографической семиотики очаговых образований щЖ.

\section{ЗАКЛЮЧЕНИЕ}

1. -ТАБ позволяет создать условия, которые повышают информативность и облегчают выполнение самой процедуры, кроме того, появляется возможность одновременно осуществлять забор цитологического материала и визуально контролировать расположение иглы с помощью УзИ одним специалистом.

Таблица 4. Анализ движений врача-оператора при тонкоигольной аспирационной биопсии на фантоме (2016-2018)

\begin{tabular}{|c|c|c|c|}
\hline $\begin{array}{c}\text { Техника выполнения/ } \\
\text { Опыт врача }\end{array}$ & $\begin{array}{c}\text { Врачом проведено >500 } \\
\text { ТАБ }(n=4)\end{array}$ & $\begin{array}{c}\text { Врачом проведено >100 } \\
\text { ТАБ }(n=3)\end{array}$ & $\begin{array}{c}\text { Врачом проведено }<100 \\
\text { ТАБ }(n=6)\end{array}$ \\
\hline $\begin{array}{l}\text { Амплитуда движения } \\
\text { по глубине, мм }\end{array}$ & До 2,0 мм & $4-5 \mathrm{Mm}$ & $6-12 \mathrm{Mm}$ \\
\hline Боковые движения, мм & До 2 мм & До 4 мм & $6-8 \mathrm{mM}$ \\
\hline $\begin{array}{l}\text { Отклонения от трассы } \\
\text { амплитуды, градусы }\end{array}$ & $3^{0}$ & $3^{0}-5^{0}$ & $10^{\circ}-20^{\circ}$ \\
\hline
\end{tabular}


Это делает представленный метод перспективным для внедрения в практику кабинетов для мини-инвазивных вмешательств при очаговой патологии щЖ.

2. Особенно это актуально для врачей с минимальным опытом работы проведения биопсий, поскольку при использовании v-ТАБ снижается риск отклонения иглы от зоны забора цитологического материала, что повышает информативность и эффективность исследования.

\section{ДОПОЛНИТЕЛЬНАЯ ИНФОРМАЦИЯ}

Источники финансирования. Работа выполнена по инициативе авторов без привлечения финансирования.
Конфликт интересов. Авторы декларируют отсутствие явных и потенциальных конфликтов интересов, связанных с содержанием настоящей статьи.

Участие авторов. Борсуков А.В. - существенный вклад в концепцию и дизайн исследования, а также внесение правки с целью повышения научной ценности статьи; Тагиль А.О. - вклад в анализ данных и интерпретацию результатов исследования, написание статьи.

Все авторы одобрили финальную версию статьи перед публикацией, выразили согласие нести ответственность за все аспекты работы, подразумевающую надлежащее изучение и решение вопросов, связанных с точностью или добросовестностью любой части работы.

\section{СПИСОК ЛИТЕРАТУРЫ| REFERENCES}

1. Борсуков А.В., Шолохов В.Н. Практическое руководство. Малоинвазивные технологии под ультразвуковой навигачией в современной клинической практике. - Смоленск; 2009. C. 28-33. [Borsukov AV, Sholohov VN. Prakticheskoe rukovodstvo. Maloinvazivnye tekhnologii pod ul'trazvukovoj navigaciej v sovremennoj klinicheskoj praktike. Smolensk; 2009. P. 28-33. (In Russ.)]

2. Бельцевич Д.Г., Ванушко В.Э., Мельниченко Г.А., и др. Клинические рекомендации Российской ассоциации эндокринологов по диагностике и лечению (много)узлового зоба у взрослых // Эндокринная хирургия. - 2016. - Т. 1. - №10. - C. 5-12. [Bel'tsevich DG, Vanushko VE, Mel'nichenko GA, et al. Russian Association of Endocrinologists Clinic Guidelines for Thyroid Nodules Diagnostic and Treatment. Endocrine Surgery. 2016;10(1):5-12. (In Russ.)]. doi: https://doi.org/10.14341/serg201615-12

3. Александров Ю.К., и др. Малоинвазивная хирургия щитовидной железы. - 2005. [Aleksandrov YuK, et al. Maloinvazivnaya hirurgiya shchitovidnoj zhelezy. 2005. (In Russ.)].

4. Сенча А.Н. Ультразвуковое исследование щитовиднойжелезы. Шаг за шагом. От простого к сложному. - М.: МЕДпрессинформ; 2019. - C. 95-119. [Sencha AN. Ultrasound examination of the thyroid gland. Step by step. From simple to complex. Moscow: Medpress-inform; 2019. P. 95-119. (In Russ.)].

5. Дедов И.И., Мельниченко Г.А., и др. Российские клинические рекомендации. Эндокринология. - М.: ГЭОТАР-Медиа; 2016. [Dedov II, Mel'nichenko GA. Rossijskie klinicheskie rekomendacii. Endokrinologiya. Moscow: GEOTAR-Media; 2016. (In Russ.)]

6. Eng CY, Quraishi MS, Bradley PJ. Management of Thyroid nodules in adult patients. Head Neck Oncol. 2010;2(1):11. doi: https://doi.org/10.1186/1758-3284-2-11

7. Александров Ю.К., и др. Эффективность стратификационных систем в диагностике узловых заболеваний щитовидной железы // Проблемы эндокринологии. - 2019. - Т. 65. №4. - C. 216-226. [Aleksandrov YK, et al. The effectiveness of risk stratification systems in diagnosis of nodular thyroid disorders. Probl Endocrinol. 2019;65(4):216-226. (In Russ.)]. doi: https://doi.org/10.14341/probl10087

8. Черников Р.А., Воробьев С.Л., Слепцов И.В. Узловой зоб (эпидемиология, методы выявления, диагностическая тактика) // Клиническая и экспериментальная тиреоидология. 2013. - T. 9. - №2. — C. 29-35. [Chernikov RA, Vorobjov SL, Slepzov IV, et al. Nodular goiter (epidemiology and diagnostics). Clinical and experimental thyroidology. 2013;9(2):29-35. (In Russ.)]. doi: https://doi.org/10.14341/ket20139229-35
9. Бельцевич Д.Г., Ванушко В.Э. Современные аспекты диагностики узлового зоба // Эндокринная хирургия. — 2014. - Т. 8. - №3. C. 5-13. [Bel'tsevich D, Vanushko V. Actual questions for thyroid nodes diagnostic. Endocrine Surgery. 2014;8(3):5-13. (In Russ.)]. doi: https://doi.org/10.14341/serg201435-13

10. Иванов Ю.В., Панченков Д.Н., Борсуков А.В., и др. Минимально инвазивная хирургия щитовидной железы. - Смоленск: Маджента; 2012. - 128 c. [Ivanov YuV, Panchenkov DN, Borsukov AV, et al. Minimal'no invazivnaya hirurgiya shchitovidnoj zhelezy. Smolensk: Madzhenta; 2012. 128 p. (In Russ.)].

11. Патент РФ на изобретение патент № R58025U1/10.08.08. Бюл. №22. Низовцев А.В., Трошин В.П. Способ взятия клеточных проб из тканей организма и устройство для его осуществления. [Patent RF na izobretenie patent № R58025U1/10.08.08. Byul. №22. Nizovcev AV, Troshin VP. Sposob vzyatiya kletochnyh prob iz tkanej organizma i ustrojstvo dlya ego osushchestvleniya. (In Russ.)].

12. Tessler F, Middleton W, Grant E. Thyroid Imaging Reporting and Data System (TI-RADS): A User's Guide. Radiology. 2018;287(3):1082-1082. doi: https://doi.org/10.1148/radiol.2018184008

13. Пранаб Д. Аспирачионная пункция тонкой иглой. Трактовка результатов и диагностические проблемы / Под ред. Н.А. Шапиро. - М.: Практическая медицина; 2015. — 109 с. [Pranab D. Aspiratsionnaya punktsiya tonkoi igloi. Traktovka rezul'tatov i diagnosticheskie problemy. Ed. by NA Shapiro. Moscow: Prakticheskaya meditsina; 2015. 109 p. (In Russ.)] doi: https://doi.org/10.5005/jp/books/12506_8

14. Воробьев С.Л. Морфологическая диагностиказаболеваний щитовидной железы. - СПб.: Коста; 2014. - 104 с. [Vorob'ev SL. Morfologicheskaya diagnostika zabolevanij shchitovidnoj zhelezy. SPb.: Kosta; 2014. - 104 p. (Russ.)].

15. Cibas E, Ali S. The 2017 Bethesda System for Reporting Thyroid Cytopathology. J Am Soc Cytopathol. 2017;6(6):217-222. doi: https://doi.org/10.1016/j.jasc.2017.09.002

16. Борсуков А.В., Амосов В.И. Ультразвуковая эластография: как делать правильно. - Смоленск; 2018. - С. 75-77. [Borsukov AV, Amosov VI. Ul'trazvukovaya elastografiya: kak delat' pravil'no. Smolensk; 2018. P. 75-77. (In Russ.)].

17. Тимофеева Л.А., Диомидова В.Н., Воропаева Л.А. Сравнение диагностической ценности лучевых методов визуализации при узловых образованиях щитовидной железы // Медицинский альманах. — 2012. - T. 4. - №63. - C. 120-123. [Timofeeva LA, Diomidova VN, Voropaeva LA. Sravnenie diagnosticheskoj cennosti luchevyh metodov vizualizacii pri uzlovyh obrazovaniyah shchitovidnoj zhelezy. Medicinskij al'manah. 2012;(4):120-123 (Russ.)].

\section{ИНФОРМАЦИЯ ОБ АВТОРАХ [AUTHORS INFO]}

*Тагиль Антон Олегович, ординатор [Anton O. Tagil, medical resident]; ORCID: 0000-0001-6400-8405; e-Library SPIN: 4740-8156; e-mail: anton.tagil95@gmail.com

Борсуков Алексей Васильевич, д.м.н., профессор [Alexey V. Borsukov, MD, PhD, prof.]; адрес: Poссия, 214006, Смоленск, ул. Фрунзе, д. 40 [address: 40 Frunze str., Smolensk, 214006, Russia]; ORCID: 0000-0003-4047-7252; e-Library SPIN: 9412-4149; e-mail: bor55@yandex.ru 


\section{ИНФОРМАЦИЯ}

Рукопись получена: 20.01.2021. Одобрена к публикации: 20.04.2021.

\section{ЦИТИРОВАТЬ:}

Борсуков А.В., Тагиль А.О. Опыт использования вакуумной тонкоигольной аспирационной биопсии под ультразвуковым контролем у пациентов с очаговой патологией щитовидной железы: пилотные результаты // Эндокринная хирургия 2020. — T. 14. — №3. - C. 13-18. doi: https://doi.org/10.14341/serg12694

\section{TO CITE THIS ARTICLE:}

Borsukov AV, Tagil AO. Experience of using vacuum fine-needle aspiration biopsy under ultrasound control in patients with focal thyroid pathology. Endocrine surgery. 2020;14(3): 13-18. doi: https://doi.org/10.14341/serg12694 